\title{
A!
}

This is an electronic reprint of the original article.

This reprint may differ from the original in pagination and typographic detail.

Martikainen, Lahja; Bertula, Kia; Turunen, Matti; Ikkala, Olli

\section{Strain Stiffening and Negative Normal Force of Agarose Hydrogels}

Published in:

Macromolecules

DOI:

10.1021/acs.macromol.0c00601

Published: $24 / 11 / 2020$

Document Version

Publisher's PDF, also known as Version of record

Published under the following license:

CC BY

Please cite the original version:

Martikainen, L., Bertula, K., Turunen, M., \& Ikkala, O. (2020). Strain Stiffening and Negative Normal Force of Agarose Hydrogels. Macromolecules, 53(22), 9983-9992. https://doi.org/10.1021/acs.macromol.0c00601

This material is protected by copyright and other intellectual property rights, and duplication or sale of all or part of any of the repository collections is not permitted, except that material may be duplicated by you for your research use or educational purposes in electronic or print form. You must obtain permission for any other use. Electronic or print copies may not be offered, whether for sale or otherwise to anyone who is not an authorised user. 


\title{
Strain Stiffening and Negative Normal Force of Agarose Hydrogels
}

\author{
Lahja Martikainen, ${ }^{*}{ }^{\perp}$ Kia Bertula, ${ }^{\perp}$ Matti Turunen, and Olli Ikkala*
}

Cite This: Macromolecules 2020, 53, 9983-9992

Read Online

ABSTRACT: Inspired by the specific strain stiffening and negative normal force phenomena in several biological networks, herein, we show strain stiffening and negative normal force in agarose hydrogels. We use both pre-strain and strain amplitude sweep protocols in dynamic rheological measurements where the gel slip was suppressed by the in situ gelation in the cross-hatched parallel plate rheometer geometry. Within the stiffening region, we show the scaling relation for the differential modulus $K \propto \sigma^{1}$, where $\sigma$ is stress. The strain at the onset of stiffening is almost constant throughout the concentration range. The gels show negative
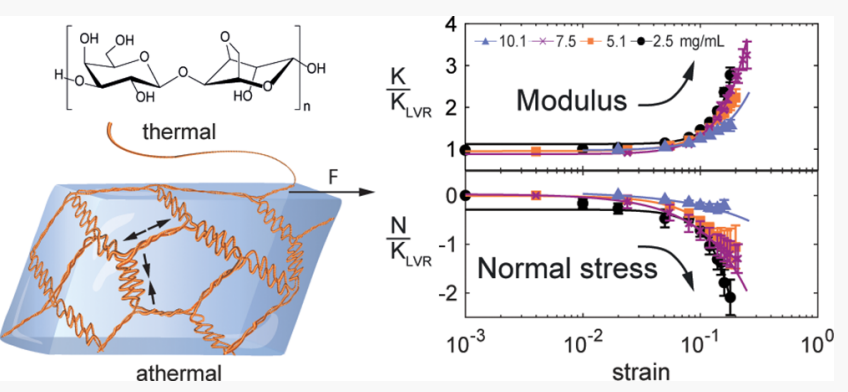
apparent normal stress difference when sheared as a result of the gel contraction. The pore size of the hydrogel is large enough to allow water to move with respect to the network to balance the pressure difference caused by the hoop stress. The rheological analysis together with scanning electron microscopy suggests that the agarose gels can be described using subisostatic athermal network models where the connectivity dictates the stiffening behavior. Therefore, the simple agarose gels appear to capture several of the viscoelastic properties, which were previously thought to be characteristic to biological protein macromolecules.

\section{INTRODUCTION}

Fibrillar hydrogels derived from biological tissues show two interesting nonlinear mechanical behaviors: strain stiffening and negative normal stress difference when shear is applied. ${ }^{1}$ Models and simulations have suggested that both phenomena arise either from the specific mechanical properties of the individual fibrils or from the network topology. The strain stiffening has commonly been reported for protein-based gels, such as actin, fibrin, and collagen, ${ }^{1,3}$ whereas it has been observed only in a few polysaccharide gels, such as in agarose, ${ }^{4}$ agar, ${ }^{5-7}$ hyaluronic acid, ${ }^{8}$ methylcellulose, ${ }^{9,10}$ pectin, ${ }^{11,12}$ and alginate, ${ }^{13}$ as well as in specific synthetic gels. ${ }^{14-17}$ Many of the strain stiffening gels show also negative normal stress difference when sheared. $1,10,13$ Their mechanical properties are typically measured with oscillatory rheology and the stiffening is seen during the amplitude strain or stress sweeps as a sudden increase in a modulus beyond a certain stress or strain value.

There are two main reasons behind the stiffening response, which can explain the behavior together or alone. If the individual fibrils are stretched beyond the Hookean limit, then they start to stiffen. Basically, the neo-Hookean model can already explain the stiffening of the elastic fibrils in tension, yet it cannot capture the strain stiffening in shear deformation at rather small strains or the negative normal force. ${ }^{18}$ In shear deformation, part of the fibrils are stretched and the others are contracted. The overall strain stiffening can be observed only if the effects of the fibrils, which are contracting and stretching, do not cancel each other out and the fibrils resist more extension than compression. Another main reason for stiffening is the topology of the network, i.e., the connectivity of the junctions. Maxwell already showed in 1864 that there should be a certain number of stiff rods connecting the junctions to form a stiff frame. ${ }^{19}$ In 1978, Calladine added a term that accounts the states of self-stresses in the network, which decreases the number of so-called floppy modes. ${ }^{20}$ If the connectivity is below the central force isostatic point (CFIP, 6 in $3 \mathrm{D}$ ), then the structure is considered as floppy, but for $\geq 6$, it will be rigid. ${ }^{21-23}$ Sharma et al. showed that this is actually a mechanical phase transition and CFIP is a critical point. ${ }^{23,24}$

Although Maxwell considered truss networks with frictionless joints, there are similarities with nearly rod-like semiflexible subisostatic athermal networks; for example, they both deform in a nonaffine way. The network can change from the floppy phase to the rigid and stiff phase either by changing the connectivity or by stretching the relatively stiff fibrils, where both reduce the degrees of freedom of the junction points of the network. ${ }^{23}$ But the network has to be mainly athermal, which basically means that the fibrils' persistence length should be significantly larger or at least at the same length scale with the mesh size of the network; otherwise, the thermal effects will be dictated. ${ }^{2,24}$ The transition from the floppy phase to

Received: March 16, 2020

Revised: August 24, 2020

Published: September 14, 2020 
a)
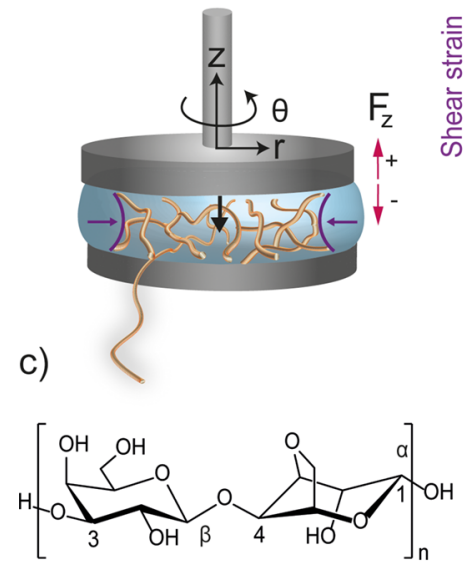

b) 0.155
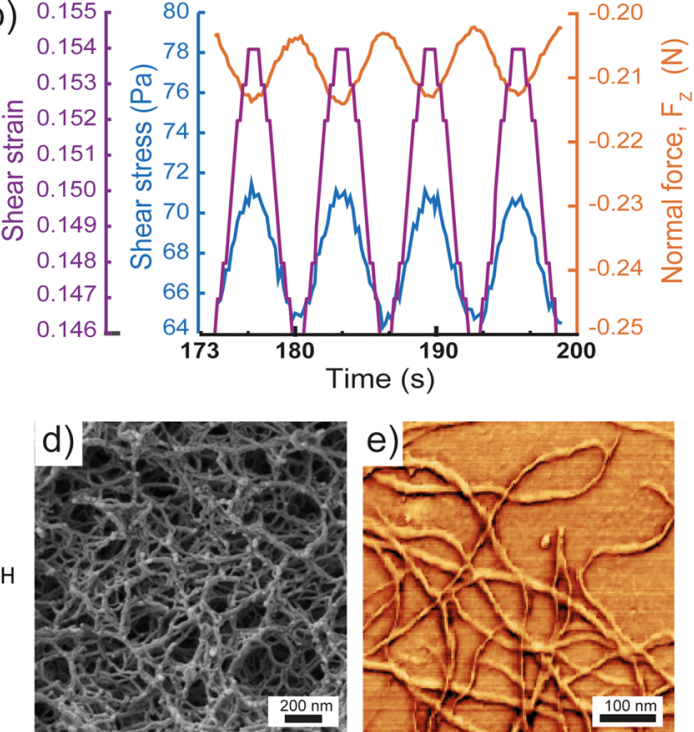

Figure 1. (a) Illustrative figure showing parallel plate geometry with a viscoelastic hydrogel and the inward contraction (violet arrows at the outer rim) of the network upon an oscillatory shear. $F_{z}$ in the right shows the present sign convention for the normal force and the arrow drawn on the upper plate illustrates the negative normal force exposed by the gel on the rheometer plates due to the sample shrinkage in the $z$-direction upon shearing. This force is compensated by the rheometer to keep the gap size constant. (b) Relationship of the normal force to the shear strain and stress for $5 \mathrm{mg} / \mathrm{mL}$ agarose gel. Note that the oscillation is imposed around a positive offset baseline. (c) Chemical structure presenting the repeat units of agarose. (d) Scanning electron microscopy image of a $10 \mathrm{mg} / \mathrm{mL}$ agarose gel. (e) Atomic force microscopy phase image of $5 \mathrm{mg} / \mathrm{mL}$ gel.

rigid phase involves also the change from bend-dominated deformations to stretch-dominated deformations. ${ }^{22,24}$ The bending deformations of the network segments are considered nonaffine, while the stretching deformations are basically affine. ${ }^{25}$ In principle, if a network is cross-linked and the fibrils are semiflexible or the fibrils are stiff and the connectivity is low, then the network will exhibit strain stiffening. When an elastic incompressible material is sheared, as shown in Figure 1a, rotation generates a so-called hoop stress, ${ }^{26}$ leading the material to expand in the $z$-direction. ${ }^{27}$ This expansion in the positive $z$-direction is seen as positive normal force and positive normal stress difference as the rheometer applies compressive normal force to the sample to keep the gap size constant. By contrast, at least three different mechanisms have been suggested to render the negative normal force where the gel shrinks in the $z$-direction upon shearing, i.e., elastic nonlinearities of the fibrils, ${ }^{1,28}$ geometrical nonlinearities, ${ }^{28}$ and fluid redistribution with respect to the network. ${ }^{26,29,30}$ Yet, they are not independent, and often, these mechanisms are connected to nonaffine deformation. ${ }^{28,31}$

Janmey et al. suggested that the negative normal stress difference is a result of nonlinear force extension of the individual fibrils. ${ }^{1}$ When a material is sheared, some of the fibrils are stretched and the others are compressed. If the fibrils completely obey Hooke's law and the deformation is affine, then such contributions would cancel each other to linear order in small strain. However, often the extension of the fibrils is energetically more costly compared with compression. If fibrils are softer for compression, then the stretched fibrils will overcompensate and the overall change in the $z$-direction is compression, which is seen as a negative normal force and stress. Cioroianu and Storm got similar results, but they also showed that the geometric nonlinearities start to have a large effect on the normal stress difference when the persistence length approaches the length scale of the mesh size. ${ }^{28}$ Meng and Terentjev concluded as well that the shearing will change the radius of the cylindrical gel sample and there will be negative normal force if the filaments are stiff or semiflexible. ${ }^{32}$

Solids, fluids, and gels are often modeled as incompressible materials. Hydrogels are polymer networks that contain a high amount of water. If a part of water is only loosely coupled to the network, then it can run through and the network may contract radially inward during the shear (Figure 1a). Therefore, the network itself is not completely incompressible. The gel contraction is seen as a decrease in normal force when shear strain or stress is increased (Figure $1 \mathrm{~b}$ and Figure S1). The pore size of agarose gels is so large that, in principle, the water can move with respect to the network (Figure 1c,d,e and Figure S2). Supported by a phenomenological two-component model, de Cagny et al. showed experimentally that the fluid embedded in a hydrogel can move in relation to the network if the pore size of the network is large enough. ${ }^{26}$ This will balance the pressure difference caused by the hoop stress, resulting in the fact that only the negative contribution from $\sigma_{\mathrm{zz}}$ is measured by the cone and plate geometry. ${ }^{26}$ The effect depends strongly on the timescale of the experiment: if the frequency is slow enough, then the fluid has time to move with respect to the network. Polyacrylamide gel typically shows a positive normal stress difference; however, a negative normal stress difference is seen if there is enough time to relax the normal stresses with fluid movement. On the contrary, fibrin gels can show an increasing normal force, instead of decreasing, as a function of shear stress if the deformation is done fast enough and the fluid remains viscously coupled. ${ }^{26}$ Similarly, Yamamoto et al. concluded based on their free energy calculations that the fluid redistribution with respect to the network will result in a negative normal force. ${ }^{29,30}$

Previously, we showed with amplitude strain sweeps that a linear polysaccharide agarose forms strain stiffening gels both in pure water and in phosphate-buffered saline (PBS) solution. ${ }^{4}$ The rheological analysis together with microscopy hinted that agarose networks can be considered as athermal 
networks with an onset of strain stiffening dictated by the connectivity of the network. Even though the slippage of the samples was minimized with in situ gelation and cross-hatched parallel plate geometry, especially the stiffer samples with higher concentration still seemed to be prone to slipping during the amplitude strain sweeps, which made the further analysis untrusworthy. ${ }^{4}$ Early slipping of the sample in the rheometer geometry will make the sample look like softening and the onset of stiffening cannot be defined; also, the normal force signal gets easily messy. There is another problem with the amplitude sweeps with constant frequency as the strain rate increases after each cycle. The strain rate has an effect as the gels are, in principle, viscoelastic, having strong time dependency on mechanical properties. ${ }^{33}$ The increasing strain rate may change the strain stiffening response remarkably. ${ }^{33}$ Using the pre-strain protocol, the problem can be avoided due to the strain rate remaining constant during the each step.

Here, we mainly use the pre-strain protocol instead of the traditional strain amplitude sweep to further minimize the slippage of agarose hydrogels and to avoid the changing strain rate. We expect this to allow more reliable studies of the scaling of the differential modulus $K$ with shear stress within the stiffening region and the strain at the onset of stiffening as a function of concentration. We also show, for the first time, that agarose gels contract when sheared and it is seen as negative normal force and apparent negative normal stress difference (Figure 1b). These, together with our previous study, give further insight into the entropic and enthalpic contributions as well the nonaffine deformation of the agarose hydrogel network.

\section{MATERIALS AND METHODS}

2.1. Materials. UltraPure Low Melting Point Agarose (lot: 000520356) was purchased from Invitrogen. Molecular weight (number average molar mass $M_{\mathrm{n}} \approx 109,000 \mathrm{~g} / \mathrm{mol}$, weight average molar mass $M_{\mathrm{w}} \approx 139,000 \mathrm{~g} / \mathrm{mol}$, and polydispersity index (PDI) = 1.28) was earlier determined for this batch by size-exclusion chromatography (SEC). ${ }^{4}$ Ultrapure Milli-Q water $(18 \mathrm{~m} \Omega)$ was used in all experiments.

2.2. Preparation of Agarose Hydrogels. Four different concentrations of agarose hydrogels were used, i.e., 2.5, 5.1, 7.5, and $10.1 \mathrm{mg} / \mathrm{mL}$. Agarose powder was first dispersed in Milli-Q water at room temperature, followed by heating to $80{ }^{\circ} \mathrm{C}$ until agarose was completely dissolved. Hot solutions were then allowed to cool down to room temperature to form a gel.

2.3. Oscillatory Rheology. Anton Paar MCR 302 equipped with RheoCompass software and Peltier plate and hood was used for the rheological measurements. We recently showed that the in situ sample preparation method with cross-hatched parallel plate geometry suppressed the effect of agarose wall slip. ${ }^{4}$ Therefore, we used such an in situ sample preparation method here as well. The hot sol was placed in prewarmed $65{ }^{\circ} \mathrm{C}$ cross-hatched parallel plate geometry. In the beginning of the measurements, the temperature was decreased to $20{ }^{\circ} \mathrm{C}$, letting the sample form a gel in situ during the measurements. To ensure the humid environment and avoid drying artifacts, water droplets were pipetted next to the parallel plate system and the Peltier hood was placed on top to seal the measurement system.

The gelling of the sample was followed by oscillation time sweep with a small amplitude of $1 \%$ and frequency of $1 \mathrm{rad} / \mathrm{s}$ for $60 \mathrm{~min}$. After the first $10 \mathrm{~min}$, the normal force was set to a constant value, 0 $\mathrm{N}$, by allowing the size of the gap to change. This will make sure that the contact between the plates and the gel stays good because the sample contracts slightly upon gelation. If the normal force is kept constant already in the beginning of the gelation process, then the gap size will first get larger as there is a small positive normal force due to the start of the gelling. Such a behavior, where the volume first increases, has been reported for the gelation of hyaluronic acid. ${ }^{8}$ After the gelation, a frequency sweep was performed with an oscillatory shear strain of $1 \%$ and angular frequency ranging from 300 to $0.1 \mathrm{rad} /$ s (Figure S3). The plateau modulus $G_{0}$ was defined as the average storage modulus from the frequency sweeps between 0.16 and 4.33 $\mathrm{rad} / \mathrm{s}$. The last step was to investigate either the strain stiffening of the gel or the effect of frequency to the normal stress difference response.

The strain stiffening was studied either by the traditional strain amplitude sweep with an oscillation frequency of $1 \mathrm{rad} / \mathrm{s}$ and strain ranging from 0.01 to $200 \%$ or alternatively using a pre-strain protocol explained as follows. In the pre-strain protocol, a sinusoidal shear strain is applied by using the sine wave generator of RheoCompass software. The sine wave generator is a part of a built-in project template in RheoCompass software, which allows the real-time recording of all the parameters during the cycles. The amplitude of the sine wave used was $1 \%$ and the offset of the wave ranged from 0 to $23 \%$. The angular frequencies were $1 \mathrm{rad} / \mathrm{s}$ for the 2.5 and $5.1 \mathrm{mg} / \mathrm{mL}$ gels, $10 \mathrm{rad} / \mathrm{s}$ for the $7.5 \mathrm{mg} / \mathrm{mL}$ gels, and 1 and $10 \mathrm{rad} / \mathrm{s}$ for the 10 $\mathrm{mg} / \mathrm{mL}$ gels. Sixteen oscillation cycles with 32 data points/cycle were recorded for each offset strain. One measurement took about $100 \mathrm{~s}$. Every other measurement was with $0 \%$ offset to let the gel rest. Each test was repeated five to eight times on each concentration. Sometimes, despite all the efforts, the gel slipped at earlier strain as agarose gels release water with time (syneresis). Those measurements were left out from the analysis. All the pre-strain results are reported as an average of four to six measurements with $95 \%$ confidence interval, unless otherwise stated.

The effect of the frequency on the normal stress difference signal was studied with the sine wave generator as well. In three different steps, each step with a larger frequency than the last one, a sine waveform strain was applied to the sample. They were applied to 5.2 $\mathrm{mg} / \mathrm{mL}$ agarose gel with $20 \%$ strain amplitude: 8 oscillation cycles (128 data points/cycle) with the frequency of $0.1 \mathrm{rad} / \mathrm{s}, 16$ oscillation cycles (64 data points/cycle) with the frequency of $1 \mathrm{rad} / \mathrm{s}$, and 16 oscillation cycles (64 data points/cycle) with the frequency of $10 \mathrm{rad} /$ s. The data were averaged over the measured oscillation cycles.

2.4. Analysis of the Rheological Data. Matlab R2019b was used for further data analysis. For the pre-strain and strain amplitude sweep measurements, the differential modulus $K=\mathrm{d} \sigma / \mathrm{d} \gamma$ is calculated, where $\sigma$ is the shear stress and $\gamma$ is the shear strain. The amplitude sweep measurements were treated similarly as previously reported. ${ }^{4}$ To obtain the differential modulus from the pre-strain protocol, the raw data needed smoothing, which was done by the means of different numerical tools in Matlab. The shear strain data were smoothed by fitting a sine wave on the data, whereas the shear stress data were first smoothed slightly with a moving average and then with a Savitzky-Golay filter. As the calculation involves division of two differences, there could be a situation where division by a very small number is done. To overcome this, the parts of the data where the sine wave is at maximum were left out of the calculation. After these steps, the differential modulus was calculated. The differential modulus was then plotted logarithmically as a function of the strain and stress amplitude and a line was fitted on the part that seemed to be the region of exponential growth.

After this, the means of the differential modulus at their respective strain values for each concentration were calculated. Confidence intervals of $95 \%$ were calculated based on the t-distribution for each point. These were then again plotted on a logarithmic scale to see a possible region of strain stiffening. An exponential fit was done within this region both with and without taking the error boundaries as a weighing factor. This same procedure was done on the normal force data.

To obtain stress, $\sigma_{\mathcal{c}}$ and strain, $\gamma_{\mathcal{c}}$ at the onset of stiffening, the same procedure was used as previously reported. ${ }^{4}$ In summary, power laws were fitted to the strain stiffening part as well as for the linear viscoelastic region (LVR). Their cross-section point was used as $\sigma_{\mathcal{c}}$ and $\gamma_{\mathrm{c}}$ was determined as a point where the $K$ had increased $10 \%$ from the average value at the LVR region.

2.5. Scanning Electron Microscopy (SEM). Scanning electron microscopy (SEM) imaging was performed with a Zeiss Sigma VP 
scanning electron microscope with an acceleration voltage of $1.5 \mathrm{kV}$. Earlier, we prepared agarose aerogels by freezing the hydrogels in liquid propane followed by lyophilization in a freeze dryer. Here, we used critical point drying to suppress the fibril aggregation, as discussed previously by Korhonen et al. ${ }^{34}$ First, water of the hydrogel was replaced by ethanol, which is miscible with $\mathrm{CO}_{2}$, via solvent exchange. Hydrogels were immersed in ethanol, which was changed three times for $30 \mathrm{~min}$ followed by overnight incubation. Samples were dried using a Bal-Tec CPD-030 and carbon dioxide as a drying agent. The sample was immersed in the ethanol-filled chamber and cooled down to $10{ }^{\circ} \mathrm{C}$, which is below the liquidification point of $\mathrm{CO}_{2}$. The chamber was flushed quickly three times followed by five times 5 min flushing with $\mathrm{CO}_{2}$ while keeping the sample immersed in the liquid. Finally, the chamber was heated to $40{ }^{\circ} \mathrm{C}$ to transform $\mathrm{CO}_{2}$ to supercritical fluid and the fluid was slowly streamed out from the chamber. Prior to the imaging, aerogel samples were coated with 10 $\mathrm{nm}$ iridium coating using a Leica EM ACE600 high vacuum sputter coater.

2.6. Analysis of the Fibril Diameter and Connectivity. The diameters of the fibrils and the connectivity were analyzed from the SEM images. ImageJ software with DiameterJ plugin was used to measure the fibril diameters. ${ }^{35}$ Original SEM images were segmented to a binary image (only black and white pixels) by Default threshold. After that, DiameterJ plugin was used to calculate diameters of the fibrils: histogram mean values with the standard deviations were collected. The fibril diameters were obtained from two different SEM images with different magnifications for all the concentrations. Connectivities were obtained manually from the SEM images and labeled in Adobe Illustrator software.

2.7. Atomic Force Microscopy (AFM). Atomic force microscopy (AFM) imaging was performed on a Veeco Dimension 5000 scanning probe microscope equipped with a Nanoscope $\mathrm{V}$ controller (Veeco, Inc., Santa Barbara, CA, USA) and HQ:NSC14/Al BS tips ( $r=8 \mathrm{~nm}$, Micromasch). Warm agarose water solution was dropcast on a plasma-treated (Gatan Solarus, model 950 plasma cleaner) silicon wafer. The excess sample was removed by washing the silicon wafer with water. The samples were gently blotted from the edge of the silicon wafer and allowed to dry before scanning by tapping mode. Gwyddion software was used for noise removal and further image analysis.

\section{THEORETICAL BASIS}

3.1. Normal Stress Difference. Taking the cylindrical coordinates of Figure 1a, the first normal stress difference $N_{1}$ and second normal stress difference $N_{2}$ are defined as follows

$$
\begin{aligned}
& N_{1}=\sigma_{\theta \theta}-\sigma_{\mathrm{zz}} \\
& N_{2}=\sigma_{\mathrm{zz}}-\sigma_{\mathrm{rr}}
\end{aligned}
$$

In parallel plate geometry, the normal stress difference $N$ can be approximated based on neo-Hookean rubber ${ }^{18}$

$$
N=N_{1}-N_{2}=\frac{4 F_{z}}{\pi R^{2}}
$$

Here, we call $N$ as the apparent normal stress difference since eq 3 actually assumes incompressibility and can be violated for hydrogels, which may become compressible. ${ }^{36}$

3.2. Entropic vs Enthalpic Deformation. The network models can be divided into thermal (entropic) and athermal (enthalpic), depending on how the undulations are considered. In both network models, the fibrils may be undulated; however, in thermal networks, the fibril undulations are sustained through thermal forces. ${ }^{37}$ The completely coiled fibrils, i.e., Gaussian chains, will respond to force extension-like thermal springs, which have an entropic modulus proportional to the temperature. ${ }^{38}$ Traditionally synthetic, permanently cross-linked chemical polymer gels are considered as thermal entropic spring networks, whereas more stiff fibrils are often considered as athermal with finite bending rigidity.

Sooner than trying to provide exact models, here, we just shortly rationalize in which length scales the entropic component of the rod-like fibrils is expected to play a significant role; for more details, see elsewhere. ${ }^{2,37,39,40}$

Here, the semiflexible fibril is nearly rod-like to the extent that we can roughly apply the classical beam model. The entropic response of semiflexible fibril comes from the thermal bending fluctuations. In contrast to the flexible polymers, the entropic stretch modulus of a semiflexible polymer is inversely proportional to the temperature. ${ }^{41,42}$ The role of thermal energy in fibril mechanics can be estimated by comparing the length of a fibril with its persistence length $l_{\mathrm{p}}$, which defines the contour length of a fibril at which significant bending fluctuation appears

$$
l_{\mathrm{p}}=\frac{\kappa}{k_{\mathrm{b}} T}
$$

where $\kappa$ is the bending stiffness of the fibril, $k_{\mathrm{b}}$ is the Boltzmann constant, and $T$ is the temperature. Fibrils are defined as semiflexible if the $l_{\mathrm{p}}$ of the fibril is around the same length scale as the fibril contour length.

To inspect whether the entropic contribution needs to take into account with semiflexible fibrils, the effective entropic and enthalpic spring constants of the beam can be compared. The effective entropic spring constant based on the review by Broedersz and MacKintosh ${ }^{2}$ is

$$
K_{\text {entropic }}=\left(90 \kappa l_{\mathrm{p}}\right) /\left(L^{4}\right)
$$

where $L$ is the length of fibril and $\kappa$ is the bending modulus, which for an elastic rod, is expressed as $\kappa=E I$, where $E$ is Young's modulus, and $I$ is the moment of inertia for a cylindrical cross section $\left(\pi R^{4}\right) / 4$, where $R$ is the radius. The universal scaling $K \propto \sigma^{3 / 2}$ observed, for example, in actin, neurofilaments, and polyisocyanopeptide hydrogels, ${ }^{2}$ is a result of entropic stretching of the biopolymers, which indicates stretching based on the thermal undulations of the fibrils.

On the other hand, the effective spring constant for stretching of an elastic beam is

$$
K_{\text {enthalpic }}=(E A) / L
$$

where $A=\pi R^{2}$ is the cross-sectional area for a cylindrical rod. $E A$ is sometimes called as the stretching modulus $\mu . K_{\text {enthalpic }}$ comes directly from Hooke's law. This enthalpic stretching is purely mechanical.

Now, we have two effective spring constants for stretching

$$
\begin{aligned}
& K_{\text {entropic }}=\left(90 E \pi R^{4} l_{\mathrm{p}}\right) /\left(4 L^{4}\right) \\
& K_{\text {enthalpic }}=\left(E \pi R^{2}\right) / L
\end{aligned}
$$

We assume that the fibril will respond primarily according to the softer effective spring constant and, therefore, the response will be entropic (thermal) only if

$$
K_{\text {entropic }}<K_{\text {enthalpic }}
$$

i.e.,

$$
(45 / 2) R^{2} l_{\mathrm{p}}<L^{3}
$$


a)

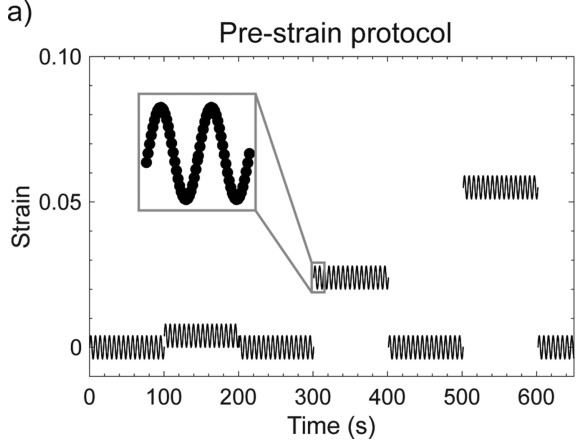

b)

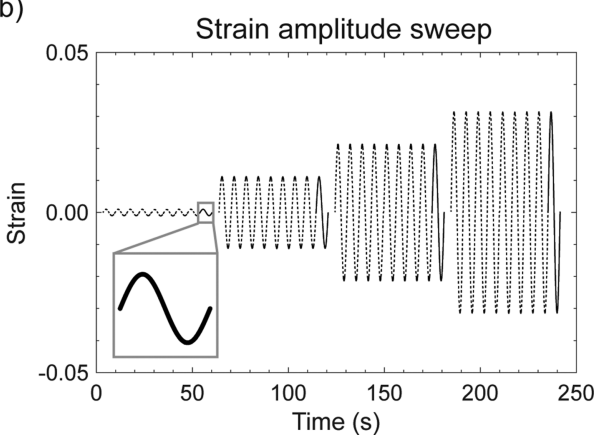

Figure 2. Difference between the pre-strain protocol and strain amplitude sweep. (a) In the pre-strain protocol, the oscillation amplitude is kept constant, while the offset strain is raised. After every step, the offset strain is returned to the zero offset. (b) During the amplitude sweep, the strain amplitude is increased, which also results in the increase in the strain rate.

If the fibril is semiflexible $\left(l_{\mathrm{p}} / L \approx 1\right)$, then $K_{\text {entropic }}$ will be the softer effective spring constant if the $L$ is approximately at least five times longer than $R$.

\section{RESULTS AND DISCUSSION}

4.1. Strain Amplitude Sweep vs the Pre-strain Protocol. In this study, we mostly use the pre-strain protocol, similar to the pre-stress protocol ${ }^{33,43}$ (Figure 2a and Figure S4), instead of the strain amplitude sweep (Figure 2b). In the dynamic amplitude sweeps, the oscillation is performed with a constant frequency (here, $1 \mathrm{rad} / \mathrm{s}$ ) and the amplitude of the cycles is increased with time. In practice, multiple oscillation cycles are first made and after reaching a constant level, one cycle or averages of a few last cycles are recorded (Figure $2 b$ ). If the amplitude increases when the frequency is kept constant, then the maximum strain rate must increase. The rate of deformation will affect how the material will perform mechanically. By contrast, with the pre-strain protocol, the change in the strain rate can be avoided. The idea is to keep the oscillation amplitude and frequency constant but just add a constant offset strain level (Figure 2a), which pushes the sample to the nonlinear regime. Here, as well, the viscoelasticity of the material results in some changes, although we always return to zero strain after each pre-strain step so that the sample can relax from the offset strain.

In principle, one can extract the same information from the data obtained by either way. The strain amplitude sweeps are suitable for soft gels with low concentrations, which do not slip easily. With the pre-strain protocol, the effect of slipping can be diminished as the measurement setup tries to keep the offset strain stable and the oscillation amplitude is small throughout the measurement. This allows us to measure higherconcentration agarose gels more reliably than previously reported. ${ }^{4}$

First, to compare pre-strain and amplitude sweep protocols, we analyzed strain stiffening and Lissajous figures with both methods. Figure 3 illustrates both cases: $5.1 \mathrm{mg} / \mathrm{mL}$ agarose hydrogels were used to illustrate the pre-strain protocol as it allows studies of medium to high concentrations without slippage and $2.5 \mathrm{mg} / \mathrm{mL}$ agarose hydrogels for strain amplitude sweeps as it is limited to low concentrations due to more pronounced slippage. Strain stiffening of the agarose gels is clearly visible regardless of the protocol. In Figure 3a,b, the differential modulus $K$ and normal stress difference are presented as a function of the shear stress. In both graphs, the normal stress difference decreases substantially and the stiffness increases almost one order of magnitude as a function
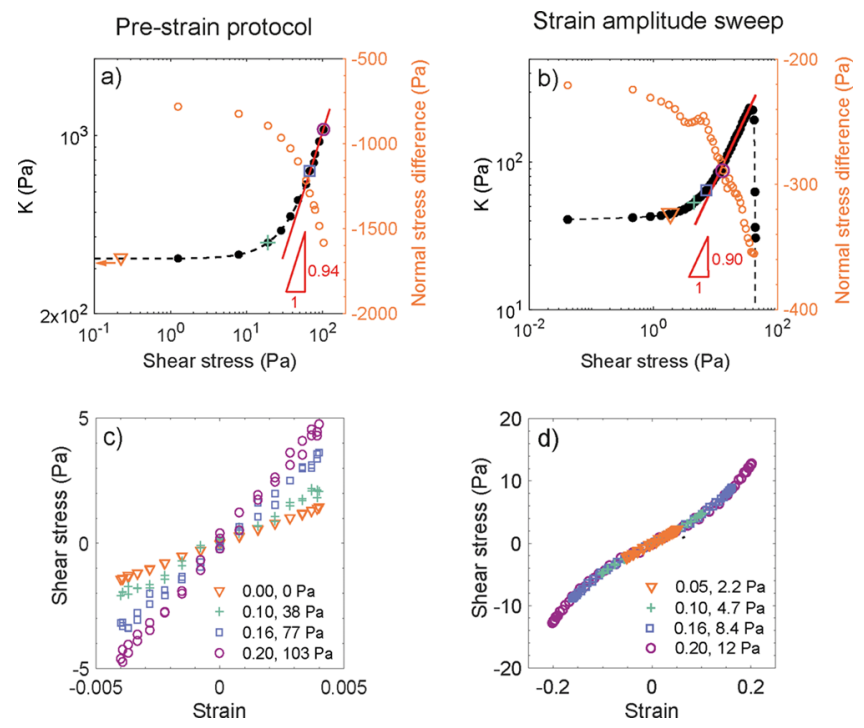

Figure 3. Difference between (a, c) the pre-strain protocol of $5.1 \mathrm{mg} /$ $\mathrm{mL}$ agarose hydrogel and $(\mathrm{b}, \mathrm{d})$ strain amplitude sweep of $2.5 \mathrm{mg} / \mathrm{mL}$ agarose hydrogel. (a, b) $\mathrm{K}$ and normal stress differences as a function of the shear stress. The black filled dots correspond to $K$ and the orange unfilled dots correspond to the apparent normal stress difference. (c) Nontypical Lissajous figures based on the pre-strain protocol. Instead of an upward bending curve, they show elastic straight lines, which is a result of the small strain amplitude of only $0.5 \%$. However, the stiffening is seen as an increase of the slopes between the different cycles. (d) Lissajous figures for the strain sweeps showing typical stiffening behaviors at large strain amplitudes within the individual cycles.

of stress. In Figure 3c,d, the Lissajous figures of selected oscillation cycles are shown. With the pre-strain protocol, the strain amplitude is sufficiently small $(0.5 \%)$, whereupon the shear stress vs strain curves seem to be completely linearly elastic (seen as straight lines in Figure 3c). Therein, the strain stiffening is not observed within a single oscillation cycle due to the small oscillation amplitude, whereas it is observed between different oscillation cycles as an increasing shear stress vs strain slope. The oscillation cycles involving the large strain amplitude sweeps show first a linear elastic behavior (up to the strain amplitude of ca. 5\%), but nonlinear behavior is observed approaching a strain amplitude of $10 \%$. Strain stiffening is identified within the individual cycles, evidenced as an upward bending of the cycles, typical for strain stiffening materials. ${ }^{44}$ 
4.2. Strain Stiffening. To investigate the strain stiffening of the agarose hydrogels in more detail, we used four different concentrations in water, i.e., $2.5,5.1,7.5$, and $10.1 \mathrm{mg} / \mathrm{mL}$, and measured the strain stiffening effect with the pre-strain protocol. In Figure 4a, the differential modulus is plotted
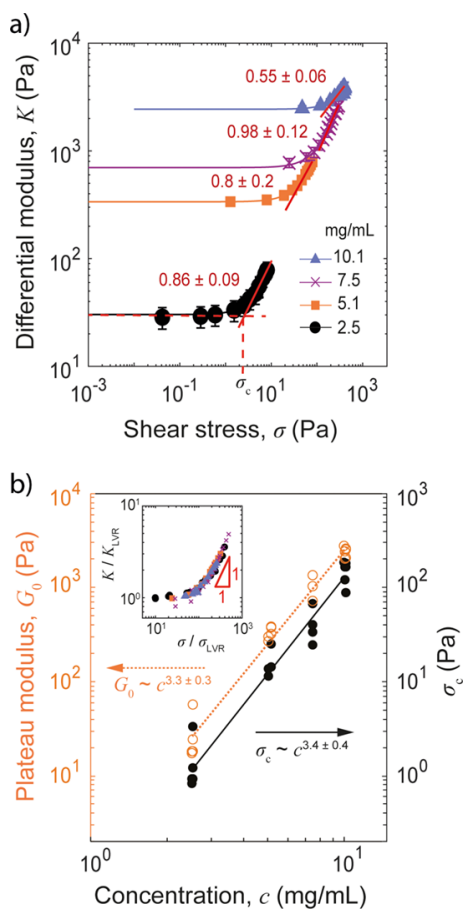

Figure 4. Differential modulus, stress at the onset of stiffening, and plateau modulus with pre-strain protocol for agarose gels. (a) $K$ as a function of $\sigma$ where the fitted power laws for the stiffening region are indicated in red. The dashed red lines show an example of defining the stress at the onset of stiffening, $\sigma_{\mathrm{c}}$, from the $K$ vs stress data. (b) Scaling of the $\sigma_{\mathrm{c}}$ and plateau modulus (the average storage modulus from the frequency sweeps) as a function of concentration. The inset shows the normalized $K$ vs $\sigma$ for each concentration, scaled by the corresponding values in the linear viscoelastic region (LVR). The slope 1.00 is indicated.

against the shear stress and a power law is fitted at the stiffening region. $K$ as a function of $\sigma$ normalized by their values at the linear viscoelastic region, $K_{\mathrm{LVR}}$ and $\sigma_{\mathrm{LVR}}$, collapses to a single master curve for different concentrations based on the pre-strain protocol (Figure $4 \mathrm{~b}$, inset). The stiffening region follows power law $K \propto \sigma^{1}$, analogous to collagen. ${ }^{45}$ Previously, we showed that the strain stiffening area of agarose was shear stress-controlled and it followed roughly power law $K \propto \sigma^{0.8}$. Therefore, it indicates that in our previous results, the lower scaling is a result of still some residual slipping despite the extensive efforts posed therein, as we expected for the strain sweeps. Typically, entropic stretching of the semiflexible fibrillar networks results in power law $K \propto \sigma^{3 / 2}$. $^{2,43}$ Here, the scaling exponent is clearly lower, which suggests that the agarose network does not behave simply like the affine entropic network. Similar scaling of $K$ with power law of approximately 1 , which reduces to $1 / 2$ either when strain is increased or if the stiffness of filaments or connectivity of the network is increased, has been shown for nonaffine athermal networks. $^{2,46,47}$

Although similar universal exponent is not recognized for athermal networks, ${ }^{46,48}$ the deviation from $3 / 2$ scaling is not enough to test whether a network is thermal or athermal. Also, athermal networks might show an initial stiffening regime with $3 / 2$ scaling. ${ }^{32,46,47}$ Therefore, simultaneous detection of the normal stress has been suggested to distinguish the difference between thermal and athermal networks. ${ }^{1,32,49,50}$ In Section 4.3 , we will present the detected negative normal forces in agarose hydrogels.

We also previously pointed out that the stress at the onset of stiffening and the plateau modulus has strong scaling with concentration (power law higher than 2 ). ${ }^{4}$ Both follow roughly the same power law as a function of concentration, i.e., approximately $c^{3 \pm 1}$. Here, the power law is just slightly stronger (around 3.35) but appropriately at the same level (Figure 4b). Similar scaling has been reported also by others. ${ }^{51}$ Also, another polysaccharide gel, i.e., hyaluronic acid, shows strong scaling $\left(c^{5.7}\right){ }^{8}$ The strong scaling might be a result from dangling or free ends, which do not participate in the elasticity of the network: at low concentrations, i.e., closer to the percolation threshold, the fraction of the free ends is higher compared with higher concentrations, which would reduce the stiffness of the gel to a lower level than it would be expected based on the concentration alone.

The strong scaling with concentration hints that the connectivity (cross-linking) might increase with concentration. $^{52,53}$ The connectivity can be estimated from the SEM images, although it is challenging due to overlapping fibrils and network structures. Earlier, we analyze the connectivity from the SEM images obtained from the samples prepared by liquid propane freeze drying. ${ }^{4}$ Now, we prepared the samples for SEM using critical point drying to compare the results (Figures S2 and S5). Similar than earlier, we show that the connectivity is mostly $z \approx 3$ but rarely $z \approx 4$ and independent of the concentration. Still, we can conclude that the estimated connectivity is below the central force isostatic point (CFIP), $z<6$. Interestingly, we noticed that critical point drying resulted in a slightly different network structure compared to liquid propane freeze drying. Fibrils seemed to be more merged together in the samples prepared by liquid propane freeze drying than in the samples prepared by critical point drying. Also, the liquid propane freeze drying affected to the network structure via the ice crystal formation, resulting in the ice crystal-shaped pores, which were not present in the samples from the critical point drying. To conclude, we showed that critical point drying is a more preferable sample preparation method than liquid propane freeze drying for the agarose hydrogels.

The differential modulus is also inspected as a function of the strain (Figure 5a,b). The stiffening of agarose gels seems to start almost at the same strain values for all concentrations. This suggests that the agarose hydrogels behave as subisostatic athermal networks, where the connectivity controls the strain at the onset of stiffening. ${ }^{23,53}$ Athermal lattice-based 2D, lattice-based 3D, and 2D Mikado models show that if the fibril rigidity is high enough, then the strain at onset of stiffening will saturate to the same value of 1 , independent of the model, connectivity, or fibril rigidity. ${ }^{46}$ Also, when the fibril rigidity approaches the so-called rope-like limit, where the network is bend-dominated, the onset strain for stiffening is independent of concentration and is dictated by the connectivity. ${ }^{46}$

4.3. Apparent Normal Stress Difference. Our previous article raised up the question whether the agarose gels show also negative normal force when sheared. ${ }^{4}$ The negative normal force means that the gel is pulling the rheometer plates 

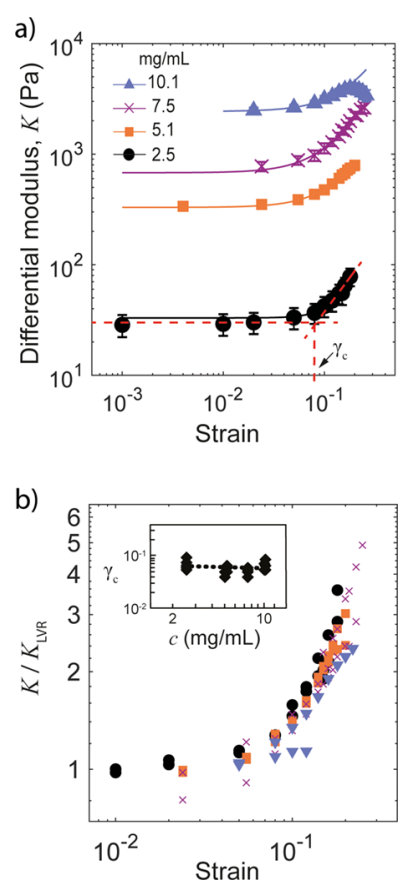

Figure 5. Differential modulus and strain at the onset of stiffening with pre-strain protocol for agarose hydrogels. (a) $\mathrm{K}$ as a function of the strain. The dashed red lines show an example of defining the strain at the onset of stiffening, $\gamma_{c}$, from the $K$ vs strain data. The solid lines are guidelines for the eyes to clarify the shape of the data. (b) Normalized $K$ vs strain shows that the behavior in the strain stiffening region does not fall into a single master curve as a function of concentration. However, the inset shows that the $\gamma_{c}$ stays approximately constant as a function of concentration.

together upon shearing. First, it should be noticed that the normal force gets negative already during the agarose gelation. Therefore, in Figure 6a, the normal force values are negative throughout the whole strain range. Hyaluronic acid ${ }^{8}$ and agar, ${ }^{7,54}$ which is a combination of agarose and agaropectin, show similar behavior during gelation. Second, the normal force becomes more negative when the strain increases; i.e., the shear strain increases the gel contraction even more. This is exactly opposite compared with the classic cases presented by Poynting and Weissenberg. ${ }^{27,55}$ Third, the absolute value of normal force is clearly larger for the higher-concentration gels. This could be also the result of internal stresses during the gelation.

To study the normal stress difference of the agarose hydrogels, we used four different concentrations in water, i.e., $2.5,5.1,7.5$, and $10.1 \mathrm{mg} / \mathrm{mL}$, and measured the effect with the pre-strain protocol. Figure $6 \mathrm{~b}$ shows the ratio between the apparent normal stress difference $N$ and shear stress $\sigma$, indicating that $|N|>|\sigma|$ for most concentrations and strain values. Also, this observation supports the athermal network behavior. $^{31,49,50}$ In particular, the appearance of the $|N / \sigma|$ overshoot has been suggested to provide experimental test to distinguish athermal effects of stiff fibrils from the thermal effects of flexible fibrils in the network. ${ }^{49,50}$ It has been concluded that the peak reflects a transition from bendingdominated regime to stretching-dominated regime, which is also typical for the athermal network. ${ }^{31}$ In a purely thermal model, the magnitude of the normal stress does not exceed the shear stress. ${ }^{1}$ Therefore, the overshoot with low concen-

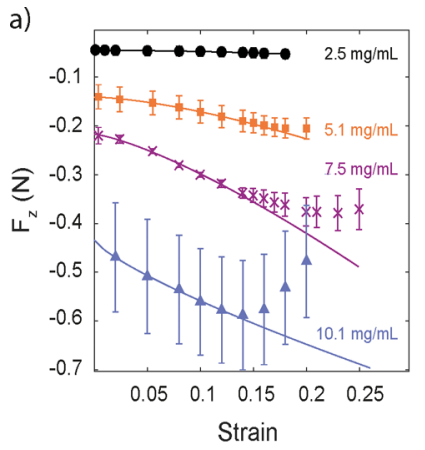

b)

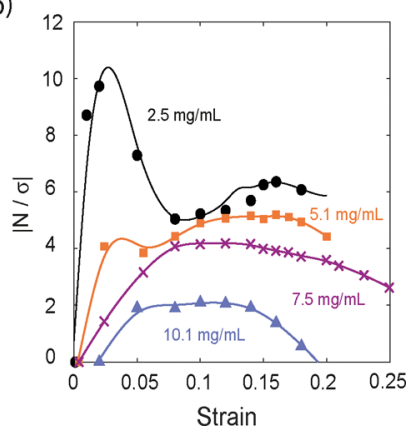

Figure 6. Normal force and normal stress difference with pre-strain protocol for agarose gels. (a) Normal force $F_{z}$ as a function of the shear strain. (b) Absolute value of the ratio between apparent normal stress difference $N$ and shear stress $\sigma$ as a function of the shear strain. The lines are just to guide the eyes.

trations, seen in Figure 6b, further confirms that the agarose fibrils are rather stiff fibrils as a part of the network.

To study whether the frequency of the deformation could change the direction of the apparent normal stress difference, we followed the method introduced by de Cagny et al. ${ }^{26}$ In three different steps, each step with a larger frequency than the last one, a sine wave form strain was applied to the sample. Three sine wave signals with different frequencies were applied to the $5.2 \mathrm{mg} / \mathrm{mL}$ agarose hydrogel. Figure $7 \mathrm{a}-\mathrm{c}$ shows the apparent normal stress difference as a function of the shear stress, where at 0.1 and $1.0 \mathrm{rad} / \mathrm{s}$, the curves form parabolas opening downward, which mean that the apparent normal stress difference changes in the even powers of shear stress. At a higher frequency of $10 \mathrm{rad} / \mathrm{s}$, the data start to be a bit noisy; however, a clear "butterfly wing" shape is observed at the upper panel, as shown previously for the fibrin gel. ${ }^{26,36}$ If the frequency is further increased, then a parabola opening upward should be a result. ${ }^{26}$ However, the data got too noisy at the higher frequencies and the upward-opened parabola could not reliably be resolved.

Figure $7 d-f$ shows the oscillation of shear stress, shear strain, and the apparent normal stress difference as a function of time. The apparent normal stress difference becomes minimum at the peaks of strain amplitudes and maximum at zero strain. At low frequencies, the stress and strain curves are almost perfectly in the same phase, but at $10 \mathrm{rad} / \mathrm{s}$, there appears to be a small phase shift between them. As the apparent normal stress difference continues to follow the strain, we get the butterfly wing shape (Figure 7c). Based on the recent studies, ${ }^{26,36}$ this would mean that water can flow through the agarose gels easily and relax the pressure difference caused by the hoop stress. As the change from downwardopening parabola to the butterfly wing shape takes place 

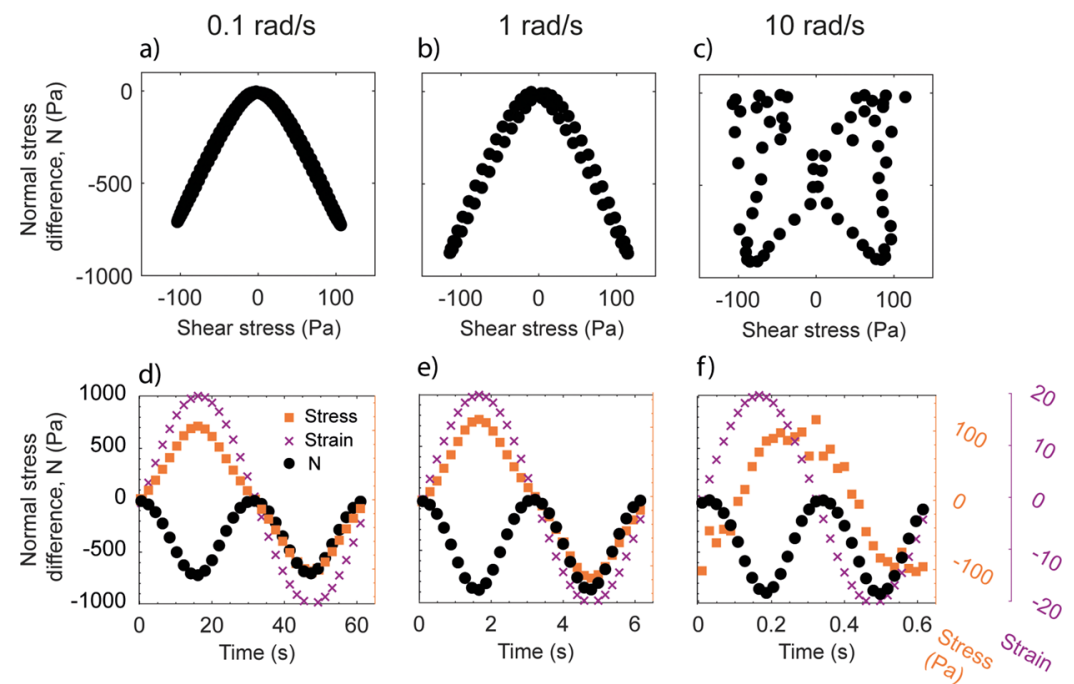

Figure 7. Effect of the frequency on the normal stress difference signal for $5.2 \mathrm{mg} / \mathrm{mL}$ agarose gels. (a-c) Apparent normal stress differences $N$ as a function of shear stress for different frequencies. $(\mathrm{d}-\mathrm{f})$ Normal stress differences, shear stresses, and shear strains as a function of time for different frequencies. Note that at 0.1 and $1 \mathrm{rad} / \mathrm{s}$, we show only every second point toward clearer images.

between 1 and $10 \mathrm{rad} / \mathrm{s}$ frequencies, the relaxation time for the $5.2 \mathrm{mg} / \mathrm{mL}$ agarose gel is in the range of $0.6-6 \mathrm{~s}$.

In agarose hydrogels, there seems to be a large fraction of water that can move quite freely. Kaneda and Iwasaki showed that compression of agarose hydrogel makes the volume decrease, which was a result of water squeezing out from the gel. ${ }^{56}$ Also, for agar organogels, the amount of free, partially structured and fully structured water has been resolved. ${ }^{57}$ In agar hydrogels, the fraction of free water was the highest. The syneresis of agar and agarose hydrogels is also related to the polymer-solvent interaction, ${ }^{58,59}$ which is believed to be an interplay between the network relaxation and solvent motion. ${ }^{59}$ All these findings suggest that there might be less polymersolvent friction than in the traditional synthetic thermal network.

The apparent normal stress does not depend on the direction of the shearing. When the shear oscillation is performed only in the positive (or negative) strain side, the normal force will stay all the time at the same negative side with $180^{\circ}$ phase difference (Figure $1 \mathrm{~b}$ ). If the oscillation is performed around the zero strain, then the normal force and apparent normal stress difference will stay at the negative side at all times so that it has its maximum absolute value at the peak of strain and minimum absolute value at zero strain or stress point (Figure $7 \mathrm{~d}$ ).

Note that in the rheological experiments, it is possible to observe both positive and negative normal forces just by overfilling or underfilling the gap between the plates, which can be easily tested with water. ${ }^{60}$ Therefore, the contact angle and surface tension are enough to change the normal force. Also, sample evaporation during the measurement will lead to an apparent negative normal force. In this study, we took special care of the fact that the gap was not underfilled; sometimes, it was just slightly overfilled in the beginning, showing a small positive normal force before gelation. The evaporation of the sample was also prevented by adding droplets of water underneath the Peltier hood.

4.4. Mesh Size vs Persistence Length. We measure the diameters of the fibrils for all the concentrations using ImageJ software (Figures S6 and S7 and Table S1). The fibril diameter was independent of the concentration being 14.1-16.8 nm, including the thin SEM metal coating. Metal coating was not reduced from the values due to the fact that the coating might not be even in each location. This is in line with the previous results, where we showed that the agarose fibril diameter is approximately $10 \mathrm{~nm} .{ }^{4}$ We also showed before that agarose fibrils have the persistence length of $1300 \pm 500 \mathrm{~nm}$ and contour length of $600 \pm 300 \mathrm{~nm}$. ${ }^{4}$ As suggested by eq 10 , the mechanical response will be more likely to be enthalpic if the mesh size is smaller than 75-100 nm and entropic if the mesh size is much larger. The SEM and AFM micrographs (Figures S2 and Figure S8) suggest that the agarose networks have dense and sparse regions, which are the result of gelation kinetics at the used concentration range. ${ }^{61,62}$ The dense parts show mesh sizes in the range of tens of nanometers. This suggests that at fibril-rich areas, the mechanical response of the agarose network would be mostly enthalpic. Still, one cannot exclude entropic response in fibril-sparse regions.

\section{CONCLUSIONS}

We investigated strain stiffening and negative normal force in agarose hydrogels using pre-strain and strain amplitude sweep protocols where the slip was suppressed by in situ gelation in the cross-hatched parallel plate rheometer geometry. Our main findings suggest that the agarose hydrogels can be described as subisostatic athermal networks. First, $K \propto \sigma^{1}$ at the strain stiffening region. Second, the strain at the onset of stiffening is almost constant throughout the concentration range. Third, a clear overshoot of the ratio between apparent normal stress difference and shear stress is observed, which reflects the change from bending-dominated mechanical response to stretching-dominated mechanical response. Last, the simple size comparison of the mesh size and persistence length by means of entropic and enthalpic spring constants suggests that the athermal response determines the mechanical behavior of agarose hydrogels. Here, we showed that the agarose hydrogels contract upon shear deformation due to the relatively large mesh size, which allows fast diffusion of water with respect to the network. The negative normal force and strain stiffening might arise from the nonaffine deformations and elastic nonlinearities of the fibrils as the agarose fibrils appear to be 
rather stiff. In conclusion, we provide experimental data supporting the idea that the individual agarose fibrils are thermal, showing semiflexibility, yet the agarose hydrogel network can be described by the subisostatic athermal model as the pore size is small compared with the persistence length of an individual fibril.

\section{ASSOCIATED CONTENT}

\section{SI Supporting Information}

The Supporting Information is available free of charge at https://pubs.acs.org/doi/10.1021/acs.macromol.0c00601.

Additional rheological data and SEM and AFM images (PDF)

\section{AUTHOR INFORMATION}

\section{Corresponding Authors}

Lahja Martikainen - Department of Applied Physics, Molecular Materials Group, Aalto University School of Science, FI-00076

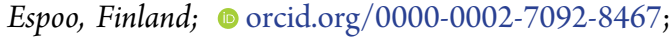
Email: lahja.martikainen@aalto.fi

Olli Ikkala - Department of Applied Physics, Molecular Materials Group, Aalto University School of Science, FI-00076 Espoo, Finland; Department of Bioproducts and Biosystems, Aalto University School of Chemical Engineering, FI-00076 Espoo, Finland; (1) orcid.org/0000-0002-0470-1889; Email: olli.ikkala@aalto.fi

\section{Authors}

Kia Bertula - Department of Applied Physics, Molecular Materials Group, Aalto University School of Science, FI-00076 Espoo, Finland; (1) orcid.org/0000-0002-7134-3591

Matti Turunen - Department of Applied Physics, Molecular Materials Group, Aalto University School of Science, FI-00076 Espoo, Finland

Complete contact information is available at:

https://pubs.acs.org/10.1021/acs.macromol.0c00601

\section{Author Contributions \\ ${ }^{\perp}$ L.M. and K.B. contributed equally to this work. All authors have given approval to the final version of the manuscript. \\ Funding \\ Academy of Finland Centre of Excellence (HYBER 2014- 2019), Business Finland (grant nos. 544/31/2015 and 2489/ 31/2017), and ERC-Advanced Grant (DRIVEN) are acknowl- edged. L.M. and K.B. acknowledge The Finnish Foundation for Technology Promotion and Walter Ahlström Foundation. L.M. acknowledges The Finnish Concordia Fund and Jenny and Antti Wihuri Foundation.}

\section{Notes}

The authors declare no competing financial interest.

\section{ACKNOWLEDGMENTS}

The authors are thankful for discussion with Professor Nonappa. We acknowledge the provision of facilities and technical support by Aalto University at OtaNano-Nanomicroscopy Center (Aalto-NMC).

\section{REFERENCES}

(1) Janmey, P. A.; McCormick, M. E.; Rammensee, S.; Leight, J. L.; Georges, P. C.; MacKintosh, F. C. Negative Normal Stress in Semiflexible Biopolymer Gels. Nat. Mater. 2007, 6, 48-51.
(2) Broedersz, C. P.; MacKintosh, F. C. Modeling Semiflexible Polymer Networks. Rev. Mod. Phys. 2014, 86, 995-1036.

(3) Storm, C.; Pastore, J. J.; MacKintosh, F. C.; Lubensky, T. C.; Janmey, P. A. Nonlinear Elasticity in Biological Gels. Nature 2005, 435, 191-194.

(4) Bertula, K.; Martikainen, L.; Munne, P.; Hietala, S.; Klefström, J.; Ikkala, O.; Nonappa. Strain-Stiffening of Agarose Gels. ACS Macro Lett. 2019, 8, 670-675.

(5) Altmann, N.; Cooper-White, J. J.; Dunstan, D. E.; Stokes, J. R. Strong through to Weak 'Sheared' Gels. J. Non-Newtonian Fluid Mech. 2004, 124, 129-136.

(6) Sousa, A. M. M.; Gonçalves, M. P. The Influence of Locust Bean Gum on Native and Alkali-Modified Agar Gels. Food Hydrocolloids 2015, 44, 461-470.

(7) Mao, B.; Bentaleb, A.; Louerat, F.; Divoux, T.; Snabre, P. HeatInduced Aging of Agar Solutions: Impact on the Structural and Mechanical Properties of Agar Gels. Food Hydrocolloids 2017, 64, 5969.

(8) Burla, F.; Tauber, J.; Dussi, S.; van der Gucht, J.; Koenderink, G. H. Stress Management in Composite Biopolymer Networks. Nat. Phys. 2019, 15, 549-553.

(9) McKee, J. R.; Hietala, S.; Seitsonen, J.; Laine, J.; Kontturi, E.; Ikkala, O. Thermoresponsive Nanocellulose Hydrogels with Tunable Mechanical Properties. ACS Macro Lett. 2014, 3, 266-270.

(10) McAllister, J. W.; Lott, J. R.; Schmidt, P. W.; Sammler, R. L.; Bates, F. S.; Lodge, T. P. Linear and Nonlinear Rheological Behavior of Fibrillar Methylcellulose Hydrogels. ACS Macro Lett. 2015, 4, 538542.

(11) Schuster, E.; Lundin, L.; Williams, M. A. K. Investigating the Relationship between Network Mechanics and Single-Chain Extension Using Biomimetic Polysaccharide Gels. Macromolecules 2012, 45, $4863-4869$.

(12) Vincent, R. R. R.; Mansel, B. W.; Kramer, A.; Kroy, K.; Williams, M. A. K. Micro-Rheological Behaviour and Nonlinear Rheology of Networks Assembled from Polysaccharides from the Plant Cell Wall. New J. Phys. 2013, 15, 035002.

(13) Hashemnejad, S. M.; Kundu, S. Strain Stiffening and Negative Normal Stress in Alginate Hydrogels. J. Polym. Sci., Part B: Polym. Phys. 2016, 54, 1767-1775.

(14) Romera, M. F.-C.; Lafleur, R. P. M.; Guibert, C.; Voets, I. K.; Storm, C.; Sijbesma, R. P. Strain Stiffening Hydrogels through SelfAssembly and Covalent Fixation of Semi-Flexible Fibers. Angew. Chem., Int. Ed. 2017, 56, 8771-8775.

(15) Kouwer, P. H. J.; Koepf, M.; Le Sage, V. A. A.; Jaspers, M.; van Buul, A. M.; Eksteen-Akeroyd, Z. H.; Woltinge, T.; Schwartz, E.; Kitto, H. J.; Hoogenboom, R.; Picken, S. J.; Nolte, R. J. M.; Mendes, E.; Rowan, A. E. Responsive Biomimetic Networks from Polyisocyanopeptide Hydrogels. Nature 2013, 493, 651-655.

(16) Tung, S.-H.; Raghavan, S. R. Strain-Stiffening Response in Transient Networks Formed by Reverse Wormlike Micelles. Langmuir 2008, 24, 8405-8408.

(17) Erk, K. A.; Henderson, K. J.; Shull, K. R. Strain Stiffening in Synthetic and Biopolymer Networks. Biomacromolecules 2010, 11, $1358-1363$.

(18) Macosko, C. W. Rheology: Principles, Measurements, and Applications; Wiley-VCH: New York, 1994.

(19) Maxwell, J. C. On the Calculation of the Equilibrium and Stiffness of Frames. Philos. Mag. J. Sci. 1864, 27, 294-299.

(20) Calladine, C. R. Buckminster Fuller's "Tensegrity" structures and Clerk Maxwell's rules for the construction of stiff frames. Int. J. Solids Struct. 1978, 14, 161-172.

(21) Broedersz, C. P.; Mao, X.; Lubensky, T. C.; MacKintosh, F. C. Criticality and Isostaticity in Fibre Networks. Nat. Phys. 2011, 7, 983-988.

(22) Feng, J.; Levine, H.; Mao, X.; Sander, L. M. Nonlinear Elasticity of Disordered Fiber Networks. Soft Matter 2016, 12, 1419-1424.

(23) Sharma, A.; Licup, A. J.; Jansen, K. A.; Rens, R.; Sheinman, M.; Koenderink, G. H.; MacKintosh, F. C. Strain-Controlled Criticality 
Governs the Nonlinear Mechanics of Fibre Networks. Nat. Phys. 2016, 12, 584-587.

(24) Sharma, A.; Licup, A. J.; Rens, R.; Vahabi, M.; Jansen, K. A.; Koenderink, G. H.; MacKintosh, F. C. Strain-Driven Criticality Underlies Nonlinear Mechanics of Fibrous Networks. Phys. Rev. E 2016, 94, 042407.

(25) Wen, Q.; Basu, A.; Janmey, P. A.; Yodh, A. G. Non-Affine Deformations in Polymer Hydrogels. Soft Matter 2012, 8, 8039-8049.

(26) de Cagny, H. C. G.; Vos, B. E.; Vahabi, M.; Kurniawan, N. A.; Doi, M.; Koenderink, G. H.; MacKintosh, F. C.; Bonn, D. Porosity Governs Normal Stresses in Polymer Gels. Phys. Rev. Lett. 2016, 117, 217802.

(27) Poynting, J. H. On Pressure Perpendicular to the Shear Planes in Finite Pure Shears, and on the Lengthening of Loaded Wires When Twisted. Proc. R. Soc. London Ser. A 1909, 82, 546-559.

(28) Cioroianu, A. R.; Storm, C. Normal Stresses in Elastic Networks. Phys. Rev. E 2013, 88, 052601.

(29) Yamamoto, T.; Masubuchi, Y.; Doi, M. Large Network Swelling and Solvent Redistribution Are Necessary for Polymer Gels to Show Negative Normal Stress. ACS Macro Lett. 2017, 6, 512-514.

(30) Yamamoto, T.; Masubuchi, Y.; Doi, M. Relaxation Dynamics of the Normal Stress of Polymer Gels. Macromolecules 2017, 50, 52085213.

(31) Shivers, J. L.; Feng, J.; Sharma, A.; MacKintosh, F. C. Normal Stress Anisotropy and Marginal Stability in Athermal Elastic Networks. Soft Matter 2019, 15, 1666-1675.

(32) Meng, F.; Terentjev, E. M. Nonlinear Elasticity of Semiflexible Filament Networks. Soft Matter 2016, 12, 6749-6756.

(33) Broedersz, C. P.; Kasza, K. E.; Jawerth, L. M.; Münster, S.; Weitz, D. A.; MacKintosh, F. C. Measurement of Nonlinear Rheology of Cross-Linked Biopolymer Gels. Soft Matter 2010, 6, 4120-4127.

(34) Korhonen, J. T.; Hiekkataipale, P.; Malm, J.; Karppinen, M.; Ikkala, O.; Ras, R. H. A. Inorganic Hollow Nanotube Aerogels by Atomic Layer Deposition onto Native Nanocellulose Templates. ACS Nano 2011, 5, 1967-1974.

(35) Hotaling, N. A.; Bharti, K.; Kriel, H.; Simon, C. G., Jr. DiameterJ: A Validated Open Source Nanofiber Diameter Measurement Tool. Biomaterials 2015, 61, 327-338.

(36) Vahabi, M.; Vos, B. E.; de Cagny, H. C. G.; Bonn, D.; Koenderink, G. H.; MacKintosh, F. C. Normal Stresses in Semiflexible Polymer Hydrogels. Phys. Rev. E 2018, 97, 032418.

(37) van Dillen, T.; Onck, P. R.; Van der Giessen, E. Models for Stiffening in Cross-Linked Biopolymer Networks: A Comparative Study. J. Mech. Phys. Solids 2008, 56, 2240-2264.

(38) Rubinstein, M.; Colby, R. H. Polymer Physics; Oxford University Press: 2003.

(39) Mechanics of Fibrous Materials and Applications. Physical and Modeling Aspects; Picu, C.; Ganghoffer, J.-F., Eds.; Springer: Cham, 2020.

(40) Pritchard, R. H.; Huang, Y. Y. S.; Terentjev, E. M. Mechanics of Biological Networks: From the Cell Cytoskeleton to Connective Tissue. Soft Matter 2014, 10, 1864-1884.

(41) Mackintosh, F. C.; Käs, J.; Janmey, P. A. Elasticity of Semiflexible Biopolymer Networks. Phys. Rev. Lett. 1995, 75, 44254428.

(42) Kroy, K.; Frey, E. Force-Extension Relation and Plateau Modulus for Wormlike Chains. Phys. Rev. Lett. 1996, 77, 306-309.

(43) Gardel, M. L.; Shin, J. H.; MacKintosh, F. C.; Mahadevan, L.; Matsudaira, P.; Weitz, D. A. Elastic Behavior of Cross-Linked and Bundled Actin Networks. Science 2004, 304, 1301-1305.

(44) Ewoldt, R. H.; Hosoi, A. E.; McKinley, G. H. New Measures for Characterizing Nonlinear Viscoelasticity in Large Amplitude Oscillatory Shear. J. Rheol. 2008, 52, 1427-1458.

(45) Licup, A. J.; Münster, S.; Sharma, A.; Sheinman, M.; Jawerth, L. M.; Fabry, B.; Weitz, D. A.; MacKintosh, F. C. Stress Controls the Mechanics of Collagen Networks. Proc. Natl. Acad. Sci. U. S. A. 2015, 112, 9573-9578.

(46) Licup, A. J.; Sharma, A.; MacKintosh, F. C. Elastic Regimes of Subisostatic Athermal Fiber Networks. Phys. Rev. E 2016, 93, 012407.
(47) Žagar, G.; Onck, P. R.; van der Giessen, E. Two Fundamental Mechanisms Govern the Stiffening of Cross-Linked Networks. Biophys. J. 2015, 108, 1470-1479.

(48) Burla, F.; Mulla, Y.; Vos, B. E.; Aufderhorst-Roberts, A.; Koenderink, G. H. From Mechanical Resilience to Active Material Properties in Biopolymer Networks. Nat. Rev. Phys. 2019, 1, 249263.

(49) Kang, H.; Wen, Q.; Janmey, P. A.; Tang, J. X.; Conti, E.; MacKintosh, F. C. Nonlinear Elasticity of Stiff Filament Networks: Strain Stiffening, Negative Normal Stress, and Filament Alignment in Fibrin Gels. J. Phys. Chem. B 2009, 113, 3799-3805.

(50) Conti, E.; MacKintosh, F. C. Cross-Linked Networks of Stiff Filaments Exhibit Negative Normal Stress. Phys. Rev. Lett. 2009, 102, 088102 .

(51) Le Goff, K. J.; Gaillard, C.; Helbert, W.; Garnier, C.; Aubry, T. Rheological Study of Reinforcement of Agarose Hydrogels by Cellulose Nanowhiskers. Carbohydr. Polym. 2015, 116, 117-123.

(52) Lin, Y.-C.; Yao, N. Y.; Broedersz, C. P.; Herrmann, H.; MacKintosh, F. C.; Weitz, D. A. Origins of Elasticity in Intermediate Filament Networks. Phys. Rev. Lett. 2010, 104, 058101.

(53) Jansen, K. A.; Licup, A. J.; Sharma, A.; Rens, R.; MacKintosh, F. C.; Koenderink, G. H. The Role of Network Architecture in Collagen Mechanics. Biophys. J. 2018, 114, 2665-2678.

(54) Mao, B.; Divoux, T.; Snabre, P. Normal Force Controlled Rheology Applied to Agar Gelation. J. Rheol. 2016, 60, 473-489.

(55) Weissenberg, K. A Continuum Theory of Rheological Phenomena. Nature 1947, 159, 310-311.

(56) Kaneda, I.; Iwasaki, S. Solvent Transportation Behavior of Mechanically Constrained Agarose Gels. Rheol. Acta 2015, 54, 437443.

(57) Boral, S.; Bohidar, H. B. Effect of Water Structure on Gelation of Agar in Glycerol Solutions and Phase Diagram of Agar Organogels. J. Phys. Chem. B 2012, 116, 7113-7121.

(58) Rinaudo, M. Main Properties and Current Applications of Some Polysaccharides as Biomaterials. Polym. Int. 2008, 57, 397-430.

(59) Boral, S.; Saxena, A.; Bohidar, H. B. Syneresis in Agar Hydrogels. Int. J. Biol. Macromol. 2010, 46, 232-236.

(60) Johnston, M. T.; Ewoldt, R. H. Precision Rheometry: Surface Tension Effects on Low-Torque Measurements in Rotational Rheometers. J. Rheol. 2013, 57, 1515-1532.

(61) San Biagio, P.L.; Bulone, D.; Emanuele, A.; Palma-Vittorelli, M.B.; Palma, M.U. Spontaneous Symmetry-Breaking Pathways: TimeResolved Study of Agarose Gelation. Food Hydrocolloids 1996, 10, 91-97.

(62) Aymard, P.; Martin, D. R.; Plucknett, K.; Foster, T. J.; Clark, A. H.; Norton, I. T. Influence of Thermal History on the Structural and Mechanical Properties of Agarose Gels. Biopolymers 2001, 59, 131144. 